\title{
Anti-Obesity Effects of Sticky Japanese Diet (SJD) Assessed by Regulations of Leptin and Adiponectin
}

\author{
Yoshiki Hirokawa ${ }^{1}$, Nobuo Izumo ${ }^{2,3}$, Masafumi Hashimoto ${ }^{3}$, Shogo Tawara ${ }^{2}$, \\ Hiroshi Mori ${ }^{1}$, Yurina Mima ${ }^{2}$, Kensuke Kuwahata ${ }^{2}$, Kazuya Watanabe ${ }^{2}$, \\ Kimiko Tsuzuki ${ }^{4}$, Yasuo Watanabe ${ }^{1,2,3^{*}}$ \\ ${ }^{1} 601$ Matano-cho, Totuka-ku, Yokohama, Kanagawa 245-0066, Japan, Lab of Functional Materials, Yokohama University of Pharmacy \\ ${ }^{2} 601$ Matano-cho, Totuka-ku, Yokohama, Kanagawa 245-0066, Japan, Lab of Food Chemistry, Yokohama University of Pharmacy \\ ${ }^{3} 601$ Matano-cho, Totuka-ku, Yokohama, Kanagawa 245-0066, Japan, General Health Medical Center, Yokohama University of Pharmacy
}

${ }^{4}$ 22-1 Tamagawa-cho, Minami-ku, Fukuoka, Fukuoka 815-8511, Japan, Tsuzuki School Group

Received: 28 March, 2019; Accepted: 9 April, 2019; Published: 16 April, 2019

*Corresponding author: Dr. Yasuo Watanabe, General Health Medical Center, Yokohama University of Pharmacy, 601 Matano-cho, Totsuka-ku, Yokohama, Kanagawa 245-0066, Japan, E-mail: yasuwat@yok.hamayaku.ac.jp

\begin{abstract}
Background: Recently, we published a book which describes about the anti-obesity efficacy of the "Nuruneba Diet (Sticky Japanese Diet; SJD)". Along with the contents of this book, the dried SJD was developed and marketed. When this marketed SJD was fed daily to obese mice, the effect of suppressing weight gain and reducing visceral fat were observed. Furthermore, we evaluated its mechanism for enhancing the leptin production. In this study, we clarify the different effects of SJD on between normal diet mice and high fat diet mice, and its mechanism was investigated focusing on adiponectin and leptin system.

Methods: 5-week-old male ICR strain mice were divided as follows: normal diet group (CE-2 group), normal diet and nuruneba (SJD) diet group (CE-2 + SJD group), high-fat diet group (HFD group), high-fat diet and Nuruneba diet it was divided into four groups (HFD + SJD group). Each group, food and water were pre-fed individually for one week and then allowed to free access to food and water for eight weeks. At the end of the treatment period, the visceral fat was collected. The triglyceride and cholesterol concentrations were determined from plasma and the expression levels of adipocytokines in visceral fat were measured by PCR.
\end{abstract}

Results: Body weight gain was observed in the HFD group, and significant suppression of body weight gain was observed in the HFD + SJD group from the third week after intake. The visceral fat was significantly increased in the HFD group compared to the CE-2 group, and significant suppression was observed in the HFD + SJD group. The effects on adipocytokines were measured for adiponectin and leptin mRNA expression. In adiponectin, the expression was significantly increased in CE-2 + SJD group compared with CE-2 group. The expression level of leptin was significantly increased in the HFD group compared to the CE-2 group, and that of leptin was significantly suppressed in the HFD + SJD.

Conclusion: These results suggest that daily intake of SJD activates adiponectin secretion under normal conditions and has an obesity preventive effect and that obesity is prevented by suppressing leptin resistance in the obese state.

Keywords: Nuruneba (Sticky Japanese Diet); Washoku (Japanese foods); normal diet; high fat diet; leptin; adiponectin; obesity, mice

List of abbreviations: SJD: Sticky Japanese Diet; HFD: High Fat Diet; CE-2: CLEA Rodent Diet CE-2

\section{Introduction}

It has been documented that most of Japanese people are belonging to non-obese types compare to the degree of obesity in European and American people. This reason can be explained that the typical Japanese food, so-called Washoku, is included by the abundance of dietary fiber. Regarding the relationship between Japanese food materials and the obese, it has been reported not only by researchers in Japan but also by overseas researchers that seaweed rich in dietary fiber is effective against obesity [1-3]. Furthermore, mushrooms are also rich in dietary fiber, and similarly, the effectiveness against obesity has been reported $[4,5]$.
On the other hand, in the recent years, an increase in obesity rate of Japanese is regarded as a problem due to widespread use of animal fat in the western food [6]. Moreover, the big problem of Japanese people is an increase of metabolic disorder due to obesity without being age including minority and full age, and it also becomes the cause of the occurrence of so-called metabolic syndrome, such as the hypertension, dyslipidemia, diabetes etc. These diseases lead to an increase in medical expenses.

In order to solve this problem, the Sticky Japanese Diet (SJD) which is the origin of Japanese food has come to be noticed. Recently, we listed several indications in the book "If you want to live a long life, you get the Sticky Japanese Diet (Japanese)" as mentioned above for consumers (Kimiko Tsuzuki, Yasuo 
Watanabe, Atsushi Ishige: Takarajima Co. Ltd). Based on this book, the commodities including 10 kinds of origin of Japanese SJD, such as root kelp (Laminariaceae), wakame (Undaria pinnatifida), agar (Generic name of raw materials: Gelidiaceae), white cloud ear (Tremella fuciformis), shiitake (Lentinula edodes), nameko (Pholiota nameko), okra (Abelmoschus esculentus), mekabu (root of Undaria pinnatifida), cut tororo (Dioscorea japonica), shimeji (Hypsizygus tessellatus) are marketed.

In the recently published paper, we found out the anti-obesity effect of this commercial product using high fat diet animals. As a result, obesity effects such as weight gain and visceral fat reduction in obese-forming mice and lowering of leptin in adipocytokines were observed [7]. Then, in this study, we examined the difference between the effect of SJD on normally ingested mice and high fat diet ingesting mice, and the mechanism was searched mainly for adiponectin and leptin.

\section{Materials \& Methods}

\section{Animal Model}

5-week-old ICR male mice was purchased from SLC (Shizuoka, Japan) individually housed in stainless steel cages with mesh bottoms at $25{ }^{\circ} \mathrm{C}$ and 40-70\% humidity, on a 12-h light (7:00 A.M.-7:00 P.M.) and dark (7:00 P.M. -7:00 A.M.) cycle. The mice were maintained with free access to water and food (standard diet commercially available) for 8 weeks in order to acclimatize.

Common food was orally administered to all mice (CE-2, Japan CLEA, Tokyo) for 1 week before they were divided into 4 groups (each group, $n=4$ ), (1) CE-2 3500mg, (2) HFD 32 (Japan CLEA, Tokyo) 3500mg, (3) CE-2 3150mg + SJD 350mg,

(4) HFD 32 3150mg + SJD 350mg under freely in taking for 8weeks.

\section{Contents of SJD}

In this experiment, we used the commercial products "SJD donated by Nack Corporation, Tokyo" which include a root kelp (Laminariaceae), wakame (Undaria pinnatifida), agar (Generic name of raw materials: Gelidiaceae), white cloud ear (Tremella fuciformis), shiitake (Lentinula edodes), nameko (Pholiota nameko), okra (Abelmoschus esculentus), mekabu (root of Undaria pinnatifida), cut tororo (Dioscorea japonica), shimeji (Hypsizygus tessellatus). We powdered this products and mixture with either the common foods or high fat foods.

\section{Food intake, body weight measurement}

Food consumption and body weight were measured during the period (every morning).

\section{Dissection}

At the end of the treatment period, the animals were fasted for 24 hours, blood and visceral fat (testicle, mesentery, perirenal) were collected.

\section{Biochemical analysis}

Container including blood added heparin was inverted and left to stand for a while. And, plasma was obtained from filtrate after centrifugal separation, 3000rpm, for 15 minutes. Plasma triglyceride concentration and cholesterol concentration were measured by using triglyceride-E-test Wako and cholesterol-Etest Wako (Wako Pure Chemical Corporation, Osaka, Japan).

\section{Adipocytokines}

Extracting total RNA was made possible by adding Isogen (Nippon Gene. Inc Tokyo) to fat tissue from each mouse and homogenized with POLYTRONPT1300D (Central trade. Inc Tokyo). It was centrifugalized after added chloroform (Nacalai Tesque. Inc, Kyoto) and filtrate was collected in another tube. This filtrate was added isopropanol (Nacalai Tesque. Inc, Kyoto) and was centrifugalized. And this sediment was collected and dissolved with sterile water. RNA concentration was measured and standardized according to Super Script VIRO cDNA Synthesis Kit Protocol (Invitrogen, Thermo Fisher Scientific, Inc). RNA was transported into cDNA after incubated under $25^{\circ} \mathrm{C}, 42{ }^{\circ} \mathrm{C}, 85^{\circ} \mathrm{C}$ for each 10 minutes, 60 minutes, 5 minutes, respectively. Then, PCR as a cycle is two times $95{ }^{\circ} \mathrm{C}$ and $60^{\circ} \mathrm{C}$ for each 10 minutes, 10 seconds, about 20 to 30 second was performed about 40 to 50 cycles to cDNA using primer and Light Cycler® 480 (F. HoffmanLa Roche, LTD., Basel, Switzerland) and the cDNA was amplified by TaqMan Prove Method (Table1).

Table 1: Primer used for each marker in Real Time PCR

\begin{tabular}{|c|c|c|c|}
\hline Gene & $\begin{array}{c}\text { Universal } \\
\text { Probe Library }\end{array}$ & Forward (Left) & Reverse (Right) \\
\hline GAPDH & $\# 9$ & AGCTTGTCATCAACGGGAAG & TTTGATGTGGGGTCTCG \\
\hline ADIPONECTIN & $\# 95$ & ACCGGCAGACAAGAGCAG & TGGTGGTACAACACCACTC \\
\hline LEPTIN & $\# 45$ & GTGGTGGCTGGTGTCAGATT & TTGATGAGTGACCAAGGTG \\
\hline
\end{tabular}




\section{Data analysis}

All results were expressed as mean and standard error. Tukey-Kramer test and t test were used for the test of significant difference, and the significance level is set to $5 \%$ or less. These data were analyzed using software Excel statistics.

\section{Results}

\section{Effects of SJD on the changes in body weight}

Although not shown in the figure, no differences in food intake were observed in each group. Figure 1 shows the change in body weight after the start of ingestion of either regular diet or high fat diet and also mixed meal with SJD diet. Graph A shows the results of weight fluctuation of the normal diet group (CE 2 group) and normal meal + SJD group (CE $2+$ SJD group). Body weight of CE2 + SJD group tended to decrease compared to CE2 group, but no significant difference was observed. Graph B shows the weight fluctuation of the high fat diet group (HFD) and the high fat diet + SJD group (HFD + SJD) HFD + SJD. A significant increase in body weight was observed from the second week on in the HFD group compared to the CE 2 group. $(\mathrm{P}<0.01)$

Furthermore, when comparing the HFD group and the HFD + SJD group, significant suppression of weight gain was observed in the HFD + SJD group compared to the HFD group from the third week after starting the feeding.
A

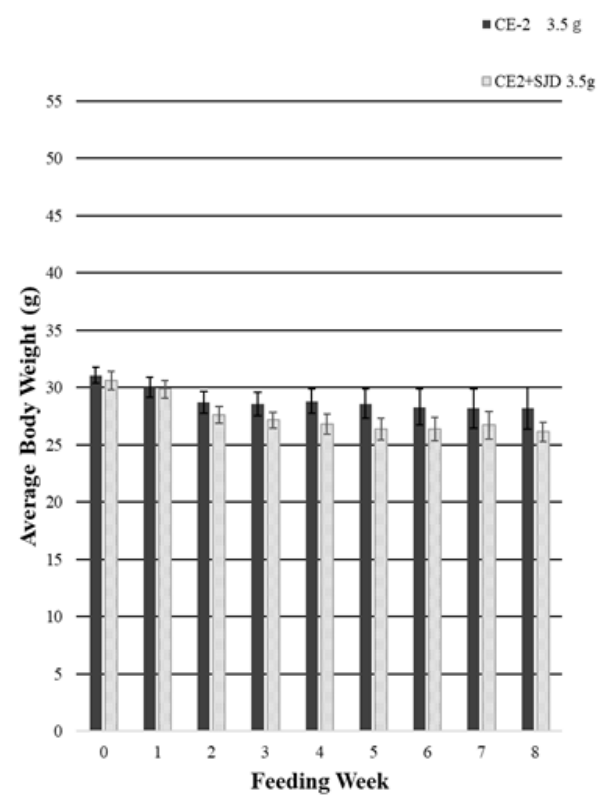

B

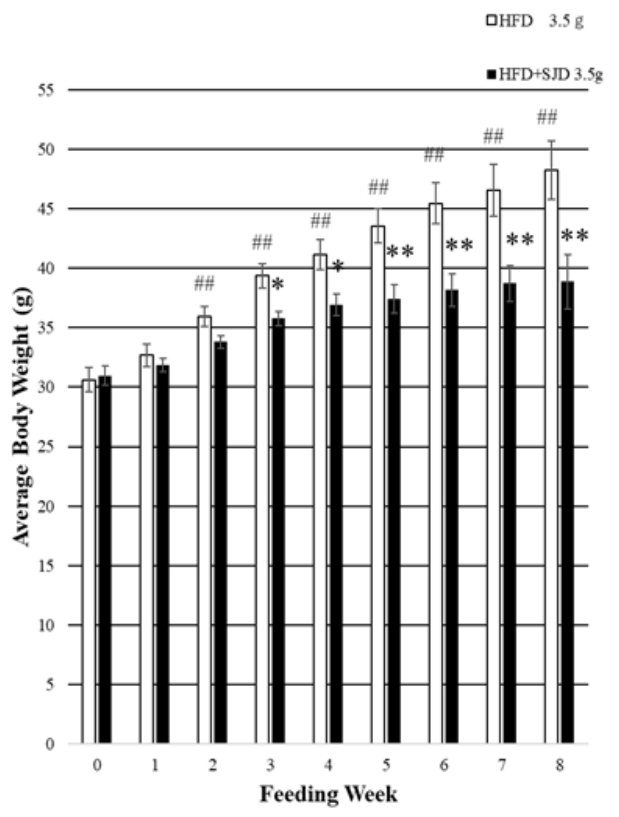

Figure 1: Effects of SJD on the changes in body weight

(A) CE2 feeding group CE2+SJD feeding group

B) HFD feeding group $\square$ HFD+SJD feeding group

Each value shows the mean \pm S.E. \#\#P $<0.01$, compared with CE2 group. ${ }^{*} \mathrm{P}<0.05,{ }^{* *} \mathrm{P}<0.01$ compared with HFD group in each week

\section{Effect of SJD on the Weight of Visceral Fat Mass}

Figure 2A shows the changes in total visceral fat of CE2 group and CE2 + SJD group, and Figure 2B shows the changes in total visceral fat in HFD and HFD + SJD group.

Visceral fat at the end of the test was $0.5 \pm 0.1 \mathrm{~g}$ in the $\mathrm{CE}$ 2 group, $0.4 \pm 0.02 \mathrm{~g}$ in the CE $2+$ SJD group, $4.9 \pm 0.5 \mathrm{~g}$ in the HFD group and $2.6 \pm 0.4 \mathrm{~g}$ in the HFD + SJD group, respectively. A significant increase in visceral fat mass was observed in the
HFD group and the HFD + SJD group as compared with the CE 2 group ( $\mathrm{P}<0.001, \mathrm{P}<0.01$, respectively). A significant decrease was observed when comparing the HFD group to the HFD + SJD group ( $\mathrm{P}<0.01)$.

From the above results, itcan be documented thataccumulation of visceral fat increases under high fat diet conditions, although the suppression of this increase can be inhibited by SJD ingestion. This inhibition reaction was not observed in the group treated with normal diet. 

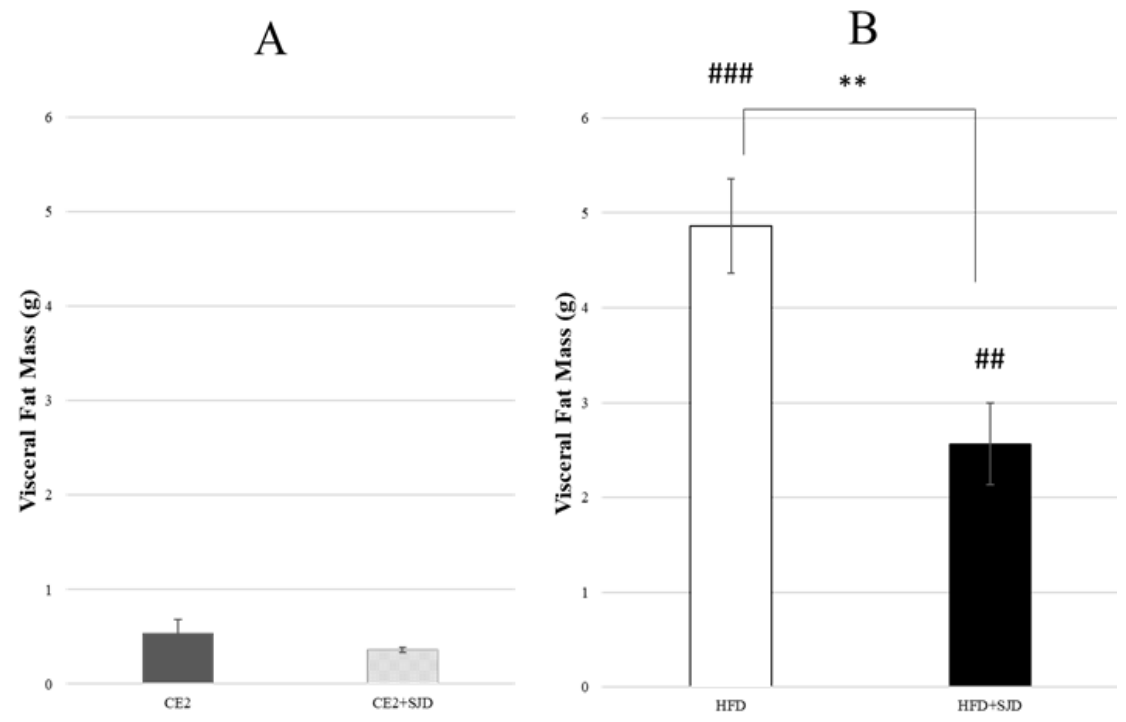

Figure 2: Effect of SJD on the weight of visceral fat mass

$\begin{array}{ll}\text { (A) CE2 feeding group } & \text { CE2+SJD feeding group } \\ \text { B) HFD feeding group } & \text { HFD+SJD feeding group }\end{array}$

Each value shows the mean \pm S.E. \#\#P $<0.01$, compared with CE2 group. ${ }^{*} \mathrm{P}<0.05,{ }^{* *} \mathrm{P}<0.01$ compared with HFD group in each week

Effect of SJD on the Plasma lipid metabolic parameters

The effects on plasma total cholesterol and plasma triglyceride levels are shown in Figure 3. Plasma total cholesterol levels were $51.8 \pm 13.6 \mathrm{mg} / \mathrm{dL}$ in CE 2 group, $32.7 \pm 8.8 \mathrm{mg} / \mathrm{dL}$ in CE $2+\mathrm{SJD}$ group, $153.6 \pm 15.9 \mathrm{mg} / \mathrm{dL}$ in HFD group and $84.2 \pm 18.7 \mathrm{mg} / \mathrm{dL}$ in HFD + SJD group. There was no significant difference between CE 2 group and CE $2+$ SJD group. In comparison between CE 2 group and HFD group, an increase in plasma cholesterol level was confirmed under high-fat food conditions ( $\mathrm{P}<0.01)$. In comparison between HFD group and HFD + SJD group, significant suppression of plasma cholesterol elevation ( $P<0.05$ ) was observed by SJD ingestion. In addition, a significant decrease in plasma cholesterol level was observed in the CE2 + SJD group compared to the HFD group ( $\mathrm{P}<0.01)$.
On the other hand, the plasma triglyceride levels were 20.6 $\pm 12.6 \mathrm{mg} / \mathrm{dL}$ in the CE 2 group, $18.9 \pm 12.9 \mathrm{mg} / \mathrm{dL}$ in the CE $2+$ SJD group, $67.8 \pm 14.5 \mathrm{mg} / \mathrm{dL}$ in the HFD group and $48.3 \pm$ $7.9 \mathrm{mg} / \mathrm{dL}$ in the HFD + SJD group. By comparing CE 2 group and HFD group, significant increase in plasma triglyceride level was confirmed under high fat dietary condition $(\mathrm{P}<0.05)$, and significant decrease in plasma triglyceride level was observed in CE2 + SJD group for HFD group $(\mathrm{P}<0.05)$. There was also a decreasing trend in the HFD + SJD group relative to the HFD group, but no significant difference was observed.

These results suggest that SJD intake significantly suppresses the elevated plasma cholesterol and slightly reduces the increases of plasma triglyceride under high fat diet conditions.
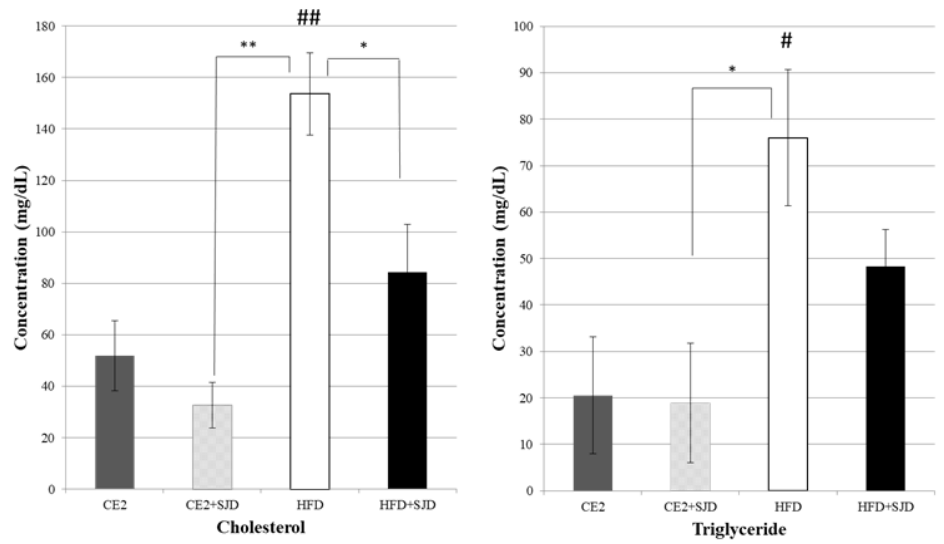

Figure 3: Effect of SJD on the cholesterol and triglyceride in plasma

Each value shows the mean \pm S.E. \#<0.05, \#\#P $<0.01$ compared with CE2 group. $* \mathrm{P}<0.05$, $* *$ P $<0.01$ compared with HFD group 


\section{Effect of SJD on mRNA expression levels of the adipo- cytokines}

The mRNA expressions of adiponectin and leptin in adipose tissue at the end of the test were measured using RT-PCR method. Gene expression was normalized using GAPDH and compared as an expression ratio with expression of CE 2 group as 1 .

The expression ratio of adiponectin was $1.5 \pm 0.1$ in the CE $2+$ SJD group, $0.2 \pm 0.05$ in the HFD group and $0.3 \pm 0.04$ in the HFD + SJD group, respectively Figure 4. Significant increase of the adiponectin expression was observed in CE2 + SJD group as compared with CE2 group $(\mathrm{P}<0.01)$. On the other hand, in the HFD group and the HFD + SJD group, there were no significant differences between the expression levels of adiponectin in two groups. However, when compared with CE 2 group, the expressions of adiponectin in both HFD group and HFD + SJD group were significantly suppressed ( $\mathrm{P}<0.01$ in both groups) In addition, there was a significant decrease in HFD group and

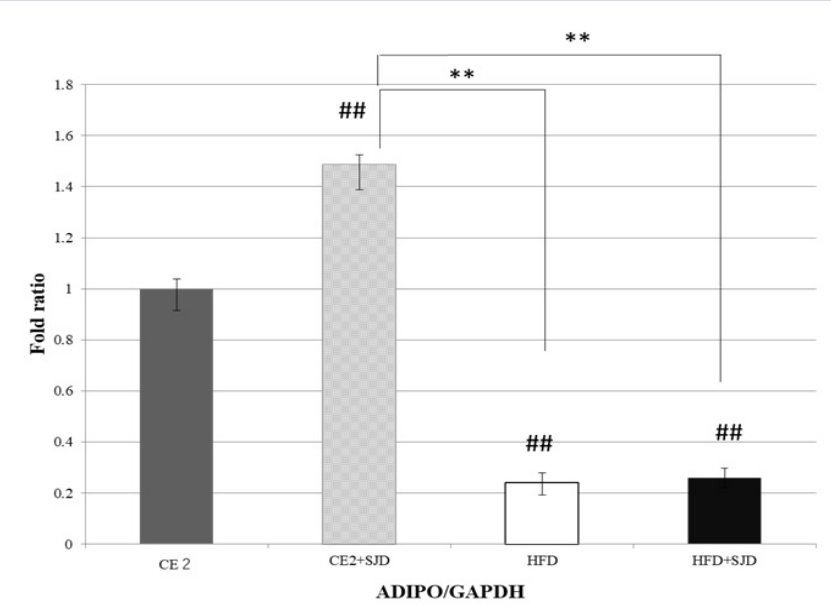

Figure 4: Effect of SJD on mRNA expression levels of the adiponectin

Each value shows the mean \pm S.E. \#\#P $<0.01$ compared with CE2 group. $* * \mathrm{P}<0.01$ compared with $\mathrm{CE} 2+\mathrm{SJD}$ group

\section{Discussion}

In this study, we used normal food intake mice and high fat diet treated mice to develop SJD (root kelp, wakame, agar, white cloud ear, shiitake, nameko, okra, mekabu, cut tororo, shimeji) on the visceral fat. As an experimental means, since individual mouse food completely by free administration, the comparative feeding amount was set at about $70 \%$ of $4.5 \mathrm{~g}$ which is the intake amount of the original mouse in the daily intake. Therefore, each mouse was observed as individual breeding, but it was in a complete state.

We have already published that commercially available SJD showed suppression of obesity growth, dose-dependently [7]. From the wakame contained in SJD, fat mass, triglyceride and cholesterol, decrease in leptin and increase in adiponectin have been reported in obese model mice $[2,8,9]$. In addition,
HFD + SJD group compared with CE2 + SJD group $(\mathrm{P}<0.01$ in both groups). From the above results, it is considered that SJD ingestion increases the amount of adiponectin in normal diet, although the production of adiponectin is suppressed in high fat diet, and its suppression could not be influenced by SJD meal.

Leptin expression ratio was $34.0 \pm 5.1$ in the CE $2+$ SJD group, $575.0 \pm 96.8$ in the HFD group and $246.4 \pm 70.2$ in the HFD + SJD group, assuming the expression rate in the CE 2 group as 1 Figure 5. A significant increase in expression was observed in the HFD group compared to the CE 2 group $(\mathrm{P}<0.01)$. Under high fat diet, HFD + SJD group showed the significant inhibition of expression compared with HFD group $(\mathrm{P}<0.01)$. In addition, there was a significant decrease in the CE2 + SJD group compared to the HFD group $(\mathrm{P}<0.01)$.

From the above results, it was revealed that expression of leptin is elevated in the high fat diet, but this increase is suppressed by ingesting SJD.

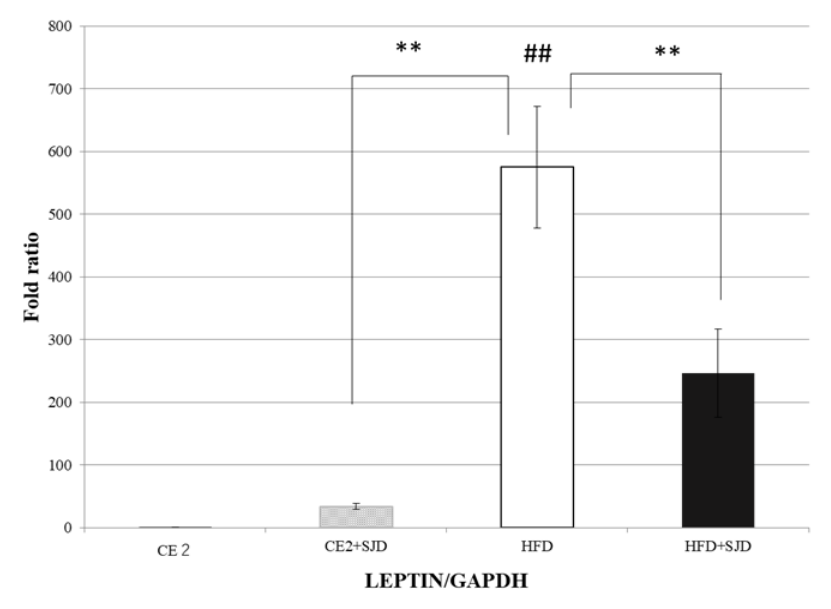

Figure 5: Effect of SJD on mRNA expression levels of the leptin Each value shows the mean \pm S.E. \#\#P $<0.01$ compared with CE2 group. ${ }^{*} \mathrm{P}<0.01$ compared with CE2+SJD group

suppression of body weight gain and decrease in the size and number of adipocytes have been reported in kelp [10-13]. Likewise, inhibition of obesity growth and increase of adiponectin have also been reported in agar and shimeji $[4,14]$. Also, reduction of leptin has been reported in Shiitake mushrooms [5].

Among adipocytokines of adipose tissue, adiponectin is a hormone secreted from normal size adipocytes, stimulating fatty acid oxidation in almost all major target tissues and secreting glucose and lipid metabolism [15-17].

In this study, administration of SJD markedly enhanced adiponectin secretion in normal mice. This result revealed that SJD promotes secretion of adiponectin, because adipocytes are normal size in normal food intake mice. In other words, it was suggested that ingesting SJD with normal diet could provide preventive effect against obesity. On the other hand, secretion 
of adiponectin decreased in adipose model because adipocytes became larger than normal size, and no effect by SJD was observed [15].

In this study, we also conducted a search on the effect of SJD on leptin, which greatly affects visceral fat increase and decrease. Leptin is a hormone from adipocytes and correlates with food intake and weight gain $[18,19]$. It also stimulates increased consumption of energy such as suppression of food intake and lipolysis by activating the leptin receptor expressed in neurons of the hypothalamus [20]. Based on the results of this experiment, despite the secretion of leptin in the HFD group than in other groups, there is no tendency for fat to decrease and it is in an obese state, so that leptin transport disorder, signal transmission disorder to the leptin receptor, etc. of leptin resistance [21]. On the other hand, in the HFD + SJD group, the leptin expression level was significantly lower than that in the HFD group, so it was considered that the increase in leptin was suppressed as the increase in fat was suppressed compared with the HFD group due to the effect of SJD.

Furthermore, blood cholesterol level and blood triglyceride level, which are two of four kinds of blood lipids, were searched. If the value is high, it is considered that dyslipidemia such as hypertriglyceridemia and hypercholesterolemia due to obesity is occurring. In the HFD group, both the cholesterol level and the triglyceride level were significantly higher than in the CE 2 group and therefore it was dietary obesity. On the other hand, in the HFD + SJD group, the increased cholesterol level was suppressed significantly, and the triglyceride value tended to decrease, so it became clear that SJD suppresses fat increase.

Overall, the results of this study and our previously reported study are summarized as follows. Commercially available SJD s including 10 kinds of root kelp, wakame, agar, white cloud ear, shiitake, nameko, okra, mekabu, cuts tororo, shimeji demonstrated to suppress obesity remarkably with a dose dependent manner and suppressed the increase of visceral fat [7]. In addition, cholesterol and triglyceride values, which are blood parameters, were reduced. In RT-PCR, gene expression level of adiponectin was not significantly different in the obesity model, but it was significantly decreased in the obesity model at the gene expression level of leptin. From blood parameters and leptin, SJD suppressed fat increase by increasing leptin efficacy without developing leptin resistance.

There was no difference in body weight and fat mass in the normal diet, but it was suggested that the SJD ingestion group was in a state where fat was easy to burn because the gene expression level of adiponectin increased.

\section{Conclusion}

The visceral fat suppression effect in commercially available SJD products was searched in the normal state and obesity formed state. As a result, it was suggested that the daily ingestion of SJD activates adiponectin secretion under normal conditions and has an effect of preventing obesity. Furthermore, it was suggested that obesity is prevented by suppressing leptin resistance in the state of obesity formation.

\section{Authors' contributions}

YH, ST, YM, KK and KW performed most of the experiments; HM took part in partial work of this research; NI and YW gave many advices during the research; YW designed the experiments; YH wrote the paper. KT gave many helpful suggestions on the writing. All authors read and approved the final manuscript.

\section{Availability of data and materials}

The datasets used and/or analyzed during the current study are available from the corresponding author on reasonable request.

\section{Ethics approval and consent to participate}

NA

\section{Clinical trial registration}

NA

\section{Acknowledgement}

The authors are thankful to Mr. Yu Kuwahara, Kazuto Homma, Jun Sakurai, and Masaya Miyazaki, students of Yokohama University of Pharmacy, for their helpful assistance while preparing the manuscript. And also, we appreciate Prof. Makoto Nakano and Dr. Kohsuke Hayamizu to their kind advices.

\section{Funding}

Annual Research Grant from Yokohama University of Pharmacy

\section{Conflict of interests}

The authors declare that they have no competing interests.

\section{References}

1. Gammone MA, D`Orazio N. Anti-obesity activity of the marine carotenoid fucoxanthin. Mar Drugs. 2015;13(4):2196-2214. doi:10.3390/md13042196

2. Grasa-López A, Miiar-García Á, Quevedo-Corona L, Paniagua-Castro N, Escaona-Cardoso G, Reyes-Maldonado E, et al. Undaria pinnatifida and Fucoxanthin Ameliorate Lipogenesis and Markers of Both Inflammation and Cardiovascular Dysfunction in an Animal Model of Diet-Induced Obesity. Mar Drugs. 2016;14(8). doi:10.3390/ md14080148

3. de Jesus Raposo MF, de Orais AM, de Morais RM. Emergent Sources of Prebiotics: Seaweeds and Microalgae. Mar Drugs. 2016;14(2). doi:10.3390/md14020027

4. Kanagasabapathy G, Kuppusamy U R, Malek S N A, Abdulla M A, Chua $\mathrm{K} \mathrm{H}$, Sabaratnam V. Glucan-rich polysaccharides from Pleurotus sajorcaju (Fr.) Singer prevents glucose intolerance, insulin resistance and inflammation in C57BL/6J mice fed a high-fat diet. BMC Complement Altern Med. 2012;12:261. doi:10.1186/1472-6882-12-261

5. Yu S, Wu X, Ferguson M, Simmen RC, Cleves MA, Simmen FA, Fang N. Diets Containing Shiitake Mushroom Reduce Serum Lipids and Serum 
Lipophilic Antioxidant Capacity in Rats. J Nutr. 2016;146(12):24912496.

6. Ministry of Health, Labor and Welfare, Outline of National Health Nutrition Survey Results, 2018.

7. Tawara S, Izumo N, Yoshikawa K, Miyazaki M, Sakurai J, Kuwahara Y, et al. Sticky and Slimy Food Materials Suppress Accumulation of Abdominal Fat in High Calorie- Diet Mice. Oyo Yakuri Pharmacometrics. 2018;95:83-90.

8. Ilavenil S, Kim DH, Vijayakumar M, Srigopalram S, Roh SG, Arasu MV, et al. Potential role of marine algae extract on 3T3-L1 cell proliferation and differentiation: an in vitro approach. Biol Res. 2016;49(1):38. doi:10.1186/s40659-016-0098-z

9. Yoshinaga K, Nakai Y, Izumi $H$, Nagaosa K, Ishijima T, Nakano T, et al. Oral Administration of Edible Seaweed Undaria Pinnatifida (Wakame) Modifies Glucose and Lipid Metabolism in Rats: A DNA Microarray Analysis. Mol Nutr Food Res. 2018;62(12):e1700828. doi:10.1002/ mnfr.201700828

10. Oh J, Lee H, Lim H, Woo S, Shin SS, Yoon M. The herbal composition GGEx18 from Laminaria japonica, Rheum palmatum, and Ephedra sinica inhibits visceral obesity and insulin resistance by upregulating visceral adipose genes involved in fatty acid oxidation. Pharm Biol. 2015;53(2):301-312. doi:10.3109/13880209.2014.917328

11. Shirosaki M, Koyama T. Laminaria japonica as a food for the prevention of obesity and diabetes. Adv Food Nutr Res. 2011;64:199-212. doi:10.1016/B978-0-12-387669-0.00015-6

12.Jang WS, Choung SY. Antiobesity Effects of the Ethanol Extract of Laminaria japonicaAreshoung in High-Fat-Diet-Induced Obese Rat. Evid Based Complement Alternat Med. 2013;2013:492807. doi:10.1155/2013/492807

13. Kim JY, Kwon YM, Kim IS, Kim JA, Yu DY, Adhikari B, et al. Effects of the Brown Seaweed Laminaria japonicaSupplementation on Serum Concentrations of IgG, Triglycerides, and Cholesterol, and Intestinal Microbiota Composition in Rats. Front Nutr. 2018;12;5:23. doi:10.3389/fnut.2018.00023
14. Hong SJ, Lee JH, Kim EJ, Yang HJ, Park JS, Hong SK. Anti-Obesity and Anti-Diabetic Effect of Neoagarooligosaccharides on High-Fat DietInduced Obesity in Mice. Mar Drugs. 2017;15(4):90. doi:10.3390/ md15040090

15. Yamauchi T, Kamon J, Waki H, Teraucih Y, Kubota N, Hara K, et al. The fat-derived hormone adiponectin reverses insulin resistance associatedwith both lipoatrophy and obesity. Nat Med. 2001;7(8):941946.

16. Nigro E, Scudiearo O, Monaco M L, Palmieri A, Mazzarella G, Costagliola $\mathrm{C}$, et al. New insight into adiponectin role in obesity and obesity-related diseases. Biomed Res Int. 2014;2014:658913. doi:10.1155/2014/658913

17. Ohashi K, Yuasa D, Shibata R, Murohara T, Ouchi N. Adiponectin as a Target in Obesity-related Inflammatory State. Endocr Metab Immune Disord Drug Targets. 2015;15(2):145-150.

18. Maffei M, Halaas J, Ravussin E, Pratley RE, Lee GH, Zhang Y, et al. Leptin levels in human and rodent: measurement of plasma leptin and ob RNA in obese and weight-reduced subjects. Nat Med. 1995;1(11):11551161.

19. Roujeau C, Jockers R, Dam J. New pharmacological perspectives for the leptin receptor in the treatment of obesity. Front Endocrinol (Lausanna). 2014;13;5:167. doi:10.3389/fendo.2014.00167

20. Harrris R B. Direct and indirect effects of leptin on adipocyte metabolism. Biochim Biophys Acta. 2014;1842(3):414-423. doi:10.1016/j.bbadis.2013.05.009

21.Zhou Y, Rui L. Leptin signaling and leptin resistance. Front Med. 2013;7(2):207-222. doi:10.1007/s11684-013-0263-5 